\title{
Ex-situ Analysis of W7-X Divertor Plasma-facing Components by Picosecond Laser Diagnostics
}

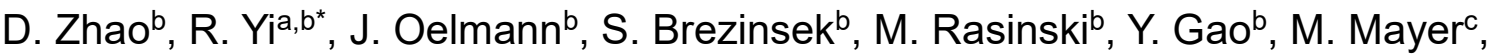 \\ C.P. Dhard ${ }^{d}$, M. Krause ${ }^{d}$, and the W7-X team ${ }^{d}$. \\ aKey Laboratory of Optoelectronic Devices and Systems of Guangdong Province, College of \\ Optoelectronic Engineering, Shenzhen University, Shenzhen 518060, P. R. China. \\ ${ }^{b}$ Forschungszentrum Jülich, Institut für Energie- und Klimaforschung - Plasmaphysik, Partner of the \\ Trilateral Euregio Cluster (TEC), 52425 Jülich, Germany \\ cMax-Planck-Institut für Plasmaphysik, 85748 Garching, Germany \\ dMax-Planck-Institut für Plasmaphysik, 17491 Greifswald, Germany \\ E-mail : r.yi@fz-juelich.de
}

\section{Abstract}

A key question of long-pulse operation in the stellarator Wendelstein 7-X (W7-X) is the net erosion of plasma-facing components (PFC) which determines W7-X plasma facing components lifetimes as well as impact on the impurity influx to the plasma. In order to judge the erosion and deposition balance at the passively cooled graphite divertor, a set of exchangeable graphite tiles with a marker layer stripes consisting of 5-10 $\mu \mathrm{m}$ C deposited on a $200 \mathrm{~nm}$ thick Mo interlayer were installed as integral part of the divertor surface and removed at the end of the first operational phase for postmortem analysis. Laser-Induced Breakdown Spectroscopy (LIBS) combined with Laser-Induced Ablation-Quadrupole Mass Spectrometry (LIA-QMS) were used for further analysis. The LIBS results show that the erosion-dominated regions are close to the location of the strike lines in standard magnetic configuration of W7-X optimised for the island divertor concept. Almost the complete $\mathrm{C} / \mathrm{Mo}$ marker layer is eroded in these regions, providing an average erosion rate of $4.3 \mathrm{~nm} / \mathrm{s}$ in the 2481 seconds plasma seconds of the campaign. In parallel, only little deposition at the edge of the divertor tiles, where Mo is observed on the bulk graphite material was found. Complementary, LIA-QMS showed a high hydrogen content on un-coated bulk graphite areas, while a lower hydrogen concentration is detected in the erosion dominated zone caused by the high surface temperature at the strike line during plasma operation.

\section{Introduction}

The world largest stellarator W7-X is successfully in operation since 2015 with limiter operations in the first campaign [1,2]. Subsequently, the uncooled graphite test 
divertor unit (TDU) was installed and W7-X operation in divertor configuration employing the island divertor concept resumed in 2017 [3, 4].

In order to study Plasma Wall Interaction (PWI) processes and the resulting material migration, 18 special divertor target elements made of fine grain graphite and coated with $\mathrm{C} / \mathrm{Mo}$ marker layers were used to study the campaign integrated erosion and deposition pattern on the divertor in W7-X operation phase 1.2A (OP 1.2A), which was the first divertor campaign in W7-X. They were exposed to $2481 \mathrm{~s}$ of hydrogen and helium plasmas in standard configuration in the first year of operation which corresponds to $65.7 \%$ of the total operational time.

Elastic Backscattering Spectrometry (EBS) and Scanning Electron Microscopy (SEM) with Focused Ion Beam (FIB) cross-sectioning were used by Dhard et al. to measure the depth profiles of $C$ and Mo on the divertor tiles [5, 6]. However, these methods are difficult to characterize the PFCs of nuclear devices in-situ. LIBS technique, currently the best known possible method to obtain accurate safety in-situ measurements of nuclear devices during operation, can be performed for elemental analysis with depth resolution of these divertor tiles. The LIBS technique has been successfully used to measure and monitor the composition on PFCs for several fusion devices [7-10]. LIA-QMS can provide simultaneously the hydrogen content in the samples with good spatial and lateral resolution avoiding the extensive use of time consuming thermal desorption spectroscopy.

In this work, $8 \mathrm{C} /$ Mo marker W7-X divertor tiles from TDU 1 upper, target element TM2h6 [6], which were exposed in OP 1.2A, were measured ex-situ by LIBS and LIAQMS. The distributions of Mo, $\mathrm{C}$ and $\mathrm{H}$ in poloidal direction and depth were obtained and used to derive the erosion and re-deposition pattern.

\section{Experimental setup and sample}

\subsection{Experimental setup}

The LIBS and LIA-QMS experimental apparatus used in this study are schematically shown in figure 1. A picosecond laser (EKSPLA, PL2241, wavelength: $355 \mathrm{~nm}$, repetition rate: $10 \mathrm{~Hz}$, pulse width: $35 \mathrm{ps}$ ) was focused on the sample surface by a lens with a focal length of $500 \mathrm{~mm}$. By focusing the laser beam to a diameter of $1 \mathrm{~mm}$ with a laser energy of $18 \mathrm{~mJ}$, the laser energy densities of $2.3 \mathrm{~J} \mathrm{~cm}^{-2}$ were achieved. The samples were mounted onto a motorized XYФ translation stage and moved in a 
straight line. A vacuum chamber and a pump were used to create an ambient pressure of $1 \times 10^{-5} \mathrm{~Pa}$ to reduce the effect from water in air. Meanwhile, a vacuometer was used to monitor the ambient pressure. For the LIBS setup, the plasma emission was coupled into an optical fibre by an optical lens and then collected by a USB spectrometer (Ocean Optics, HR2000, wavelength range: 350$800 \mathrm{~nm}$ ). For the LIA-QMS setup, a quadrupole mass spectrometer (Pfeiffer HiQuad QMS 700) is used to determine the composition of residual gases.

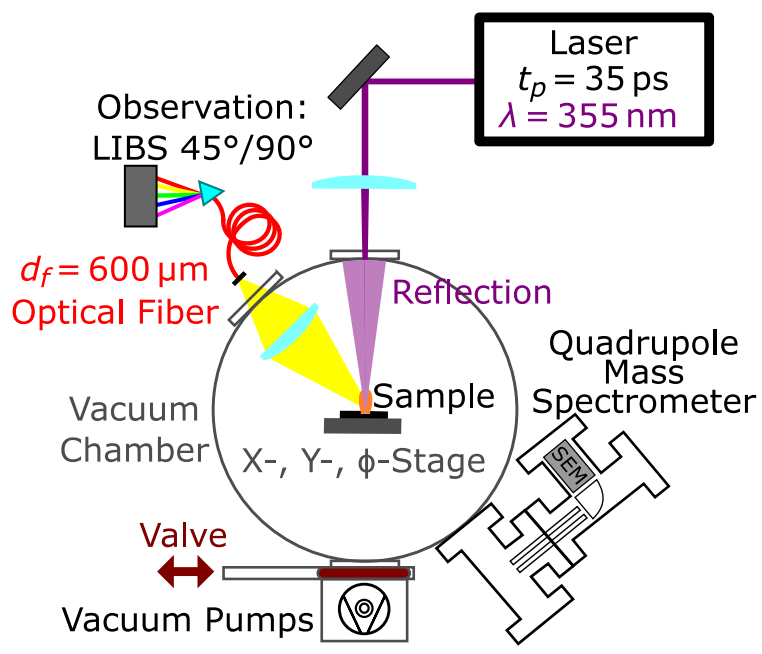

Figure 1. Schematic of the setup for simultaneous LIBS \& LIA-QMS analysis of W7-X limiter tiles in vacuum [8].

\subsection{Samples}

During OP 1.2A of W7-X, in order to quantify the erosion and deposition of $\mathrm{C}$ on the divertor, a set of marker tiles was installed and removed at the end of OP1.2A $[4,5]$. As shown in figure 2(a), a series of eight samples (TM2h6, marked with red dot line) in poloidal direction were chosen for analysis. The width of the divertor tiles is 75 $\mathrm{mm}$. The samples were measured with a lateral resolution of $5 \mathrm{~mm}$ (first crater $2 \mathrm{~mm}$ from edge,) laser craters were marked by red points on the sample in figure 2(b), all the samples contained 15 laser craters, except for samples E012 and A012 which have 6 and 13 craters, respectively. These tiles were partially coated (stripe area $63 \times 25 \mathrm{~mm}$ ) with a $200 \mathrm{~nm}$ thick Mo layer and with 5-10 $\mu \mathrm{m} \mathrm{C}$ deposited on the Mo layer (using the CMSII method [11]), as shown figure 2(c). In order to achieve information about the material composition in the depth multiple laser pulses were used at a single measurement point. To ablate the whole marker layer - in case it is 
fully intact - from the samples, on each measurement point 220 laser pulses were used for subsequent ablation and spectra recording of every single ablation.

(a)
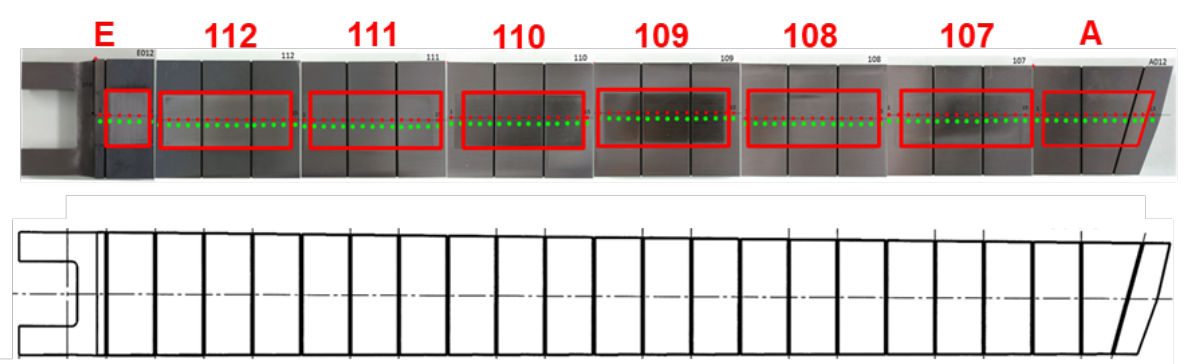

(b)

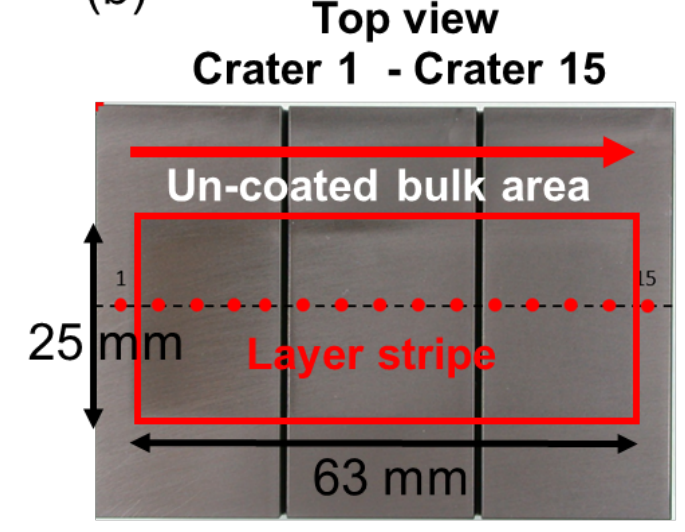

(c)

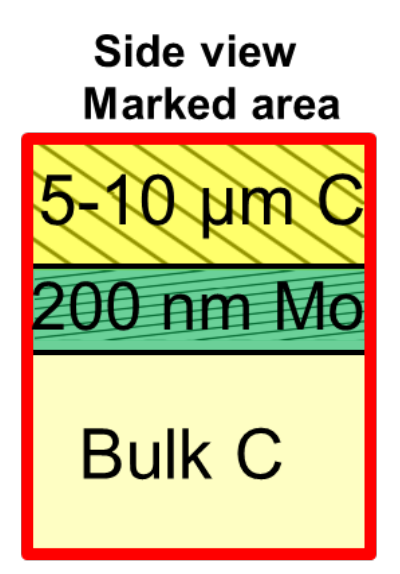

Figure 2. a) Photo of the PWI divertor tiles exposed in OP 1.2A, the dots show the spatial resolved LIBS measurement positions along the poloidal direction. b) Laser ablation craters in one sample, all the samples contained 15 craters, except for sample $E$ and $A$ which have 6 and 13 craters, respectively. c) Schematic representation of the layer structure of the marked area.

\section{Results and analysis}

\subsection{Results of LIBS}

Typical LIBS spectra of the marker layer and of bulk graphite are shown in figure 3. The blue, green and red lines represent the spectra from the first graphite layer, Mo layer and the bulk graphite area, respectively. The signals of Mo I at $550.68 \mathrm{~nm}, \mathrm{Na}$ I at $588.99 \mathrm{~nm}, \mathrm{HI}$ at $656.31 \mathrm{~nm}, \mathrm{C} \mathrm{II}$ at $657.81 \mathrm{~nm}$ and $\mathrm{O} I$ at $777.35 \mathrm{~nm}$ can be observed clearly in the spectra. The spectral intensity of these elements varies in the different areas probed by the laser beam which was limited by the dynamic range of 
the detection system. Note that Mo was in general not detectable on the surface of the uncoated graphite areas except for some minor local areas in toroidal direction near the strike-line where Mo was locally prompt re-deposited though one operates in the net erosion zone.

The spectral intensity of Mo I emission varies in the different laser pulse numbers due to the layer structure of the marker areas. Therefore, the signal of Mo can be used to determine the erosion and redeposition conditions of the divertor tiles for both $\mathrm{C}$ (top surface) and Mo (interlayer).

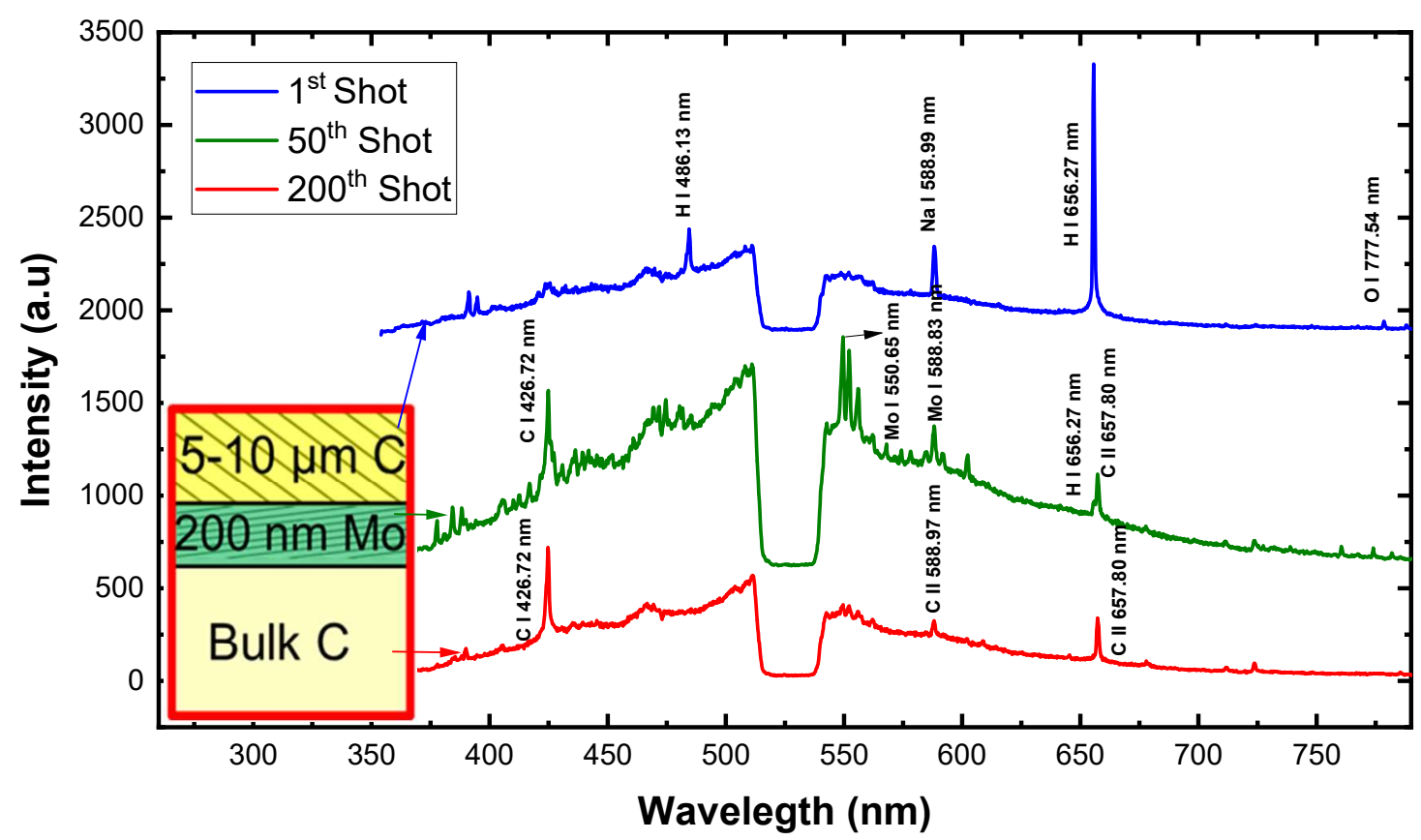

Figure 3. Typical LIBS spectra of the marker layer and of the bulk graphite. Mo, $\mathrm{H}, \mathrm{C}$ and $O$ lines can be observed. Different spectra were obtained from different layers. Due to a laser notch filter (532 nm laser filter), a dip appeared in the region from 520$540 \mathrm{~nm}$. Continuum line emission appeared from the long exposure time of the ungated detector array.

Figure 4(a) shows the Mo signal (background removed by baseline) distribution of different laser pulses in crater 9 of sample \#107. The signal of Mo increases from the $10^{\text {th }}$ laser pulse and reaches its maximum value at the $46^{\text {th }}$ laser pulse, then its intensity decreases until the $126^{\text {th }}$ pulse. The appearance and disappearance of the Mo signal can be obtained from figure 4(a), which represents the upper and lower surface of the Mo layer, respectively. Figure 4(b) shows the same distribution of Mo 
of the whole sample \#107 in poloidal direction (15 craters), the blue dotted line region shows that the signals of Mo exist in the first few laser pulses in crater $15^{\text {th }}$ (uncovered area), which represents redeposition in this cater.

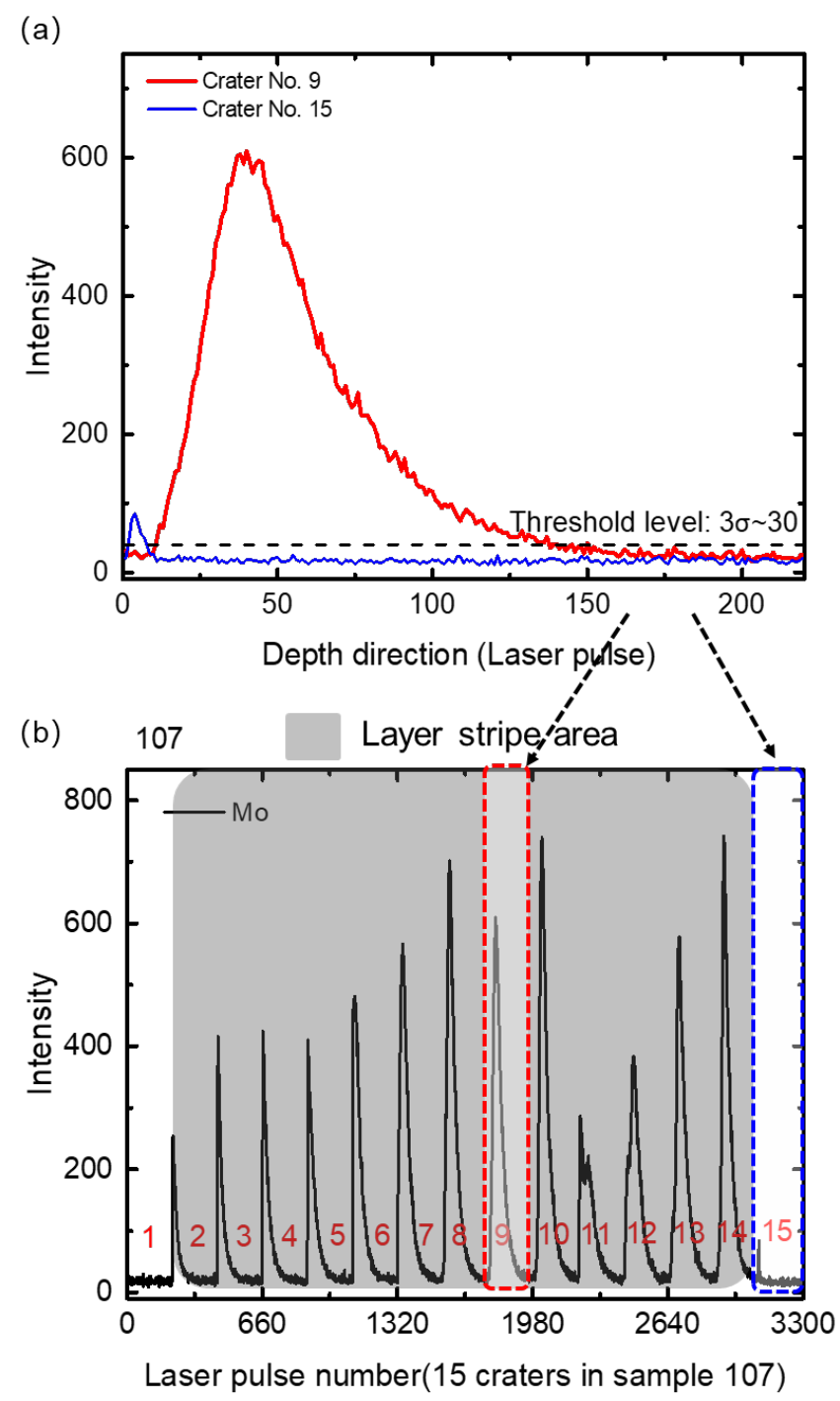

Figure 4. (a) Mo signal distribution of different laser pulses in crater No. 9 and 15 on sample \#107, the black line represents the background threshold of the signal, while the spectral intensity is higher than the line, it is considered as an effective signal. (b) The Mo signal distribution of the whole sample (contains 15 craters in one line). The blue dotted line shows that the signal of Mo exists in the first few laser pulses of crater No. 15, which represents redeposition at this point

To map the erosion region we obtained the appearance and disappearance of the laser pulse numbers of the Mo signal for all the 8 samples. The judgement was 
based on the background threshold in Figure 4(a). As shown in figure 5, the Mo signal appeared at different laser pulse numbers in different sample positions. On the right part of the divertor such as sample \#112, the signal appeared after the $35^{\text {th }}$ laser pulse, but in the right part of sample \#108 and the left part of sample \#107, the Mo signal appeared almost at the 1st laser pulse. This means, that the graphite layer on top of the Mo layer has been erased totally in OP 1.2A. Meanwhile, the Mo signal disappeared at different laser pulses in different positions as well. In sample \#112, it disappeared after the 190th laser pulse. The signal existed for more than 150 laser pulses, which means the layer of Mo exist in the 150 pulses. However, the early disappearance of Mo signal happened in samples \#108 and \#107, which indicated that not only the $\mathrm{C}$ layer but also the Mo layer has been erased as well in this region. Therefore, the region around sample \#108 and \#107 were called the high erosion region (red dotted lines region). Moreover, the ablation rate of pulse laser was measured, it is $108 \mathrm{~nm} /$ pulse for $C$ layer and $43 \mathrm{~nm} /$ pulse for Mo layer. The averaged depth of the C/Mo marker layer on TDU before plasma explosion is calculated to about $10.7 \mu \mathrm{m}$ by LIBS method in this work. The average erosion rate of the C/Mo maker is about $4.3 \mathrm{~nm} / \mathrm{s}$ in OP1.2A campaign in the erosion dominated region.

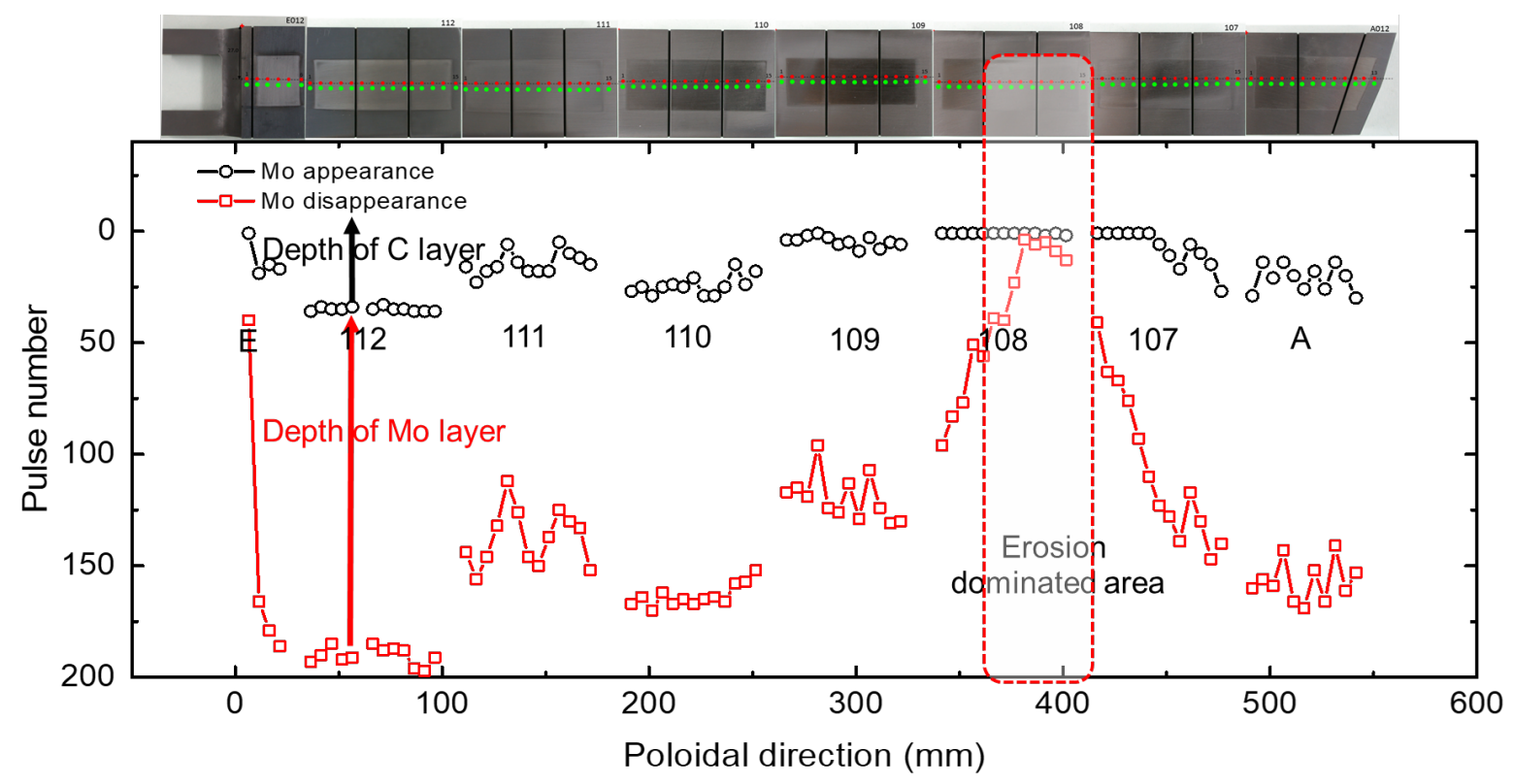

Figure 5. The erosion condition of the whole sample series. The pulse number of the black hollow point represents the thickness of the $C$ layer, the pulse number between black and red hollow point represents the thickness of the Mo layer. Due to the 
narrow gap between the black and red hollow point, the region marked by red box is identified as with high erosion where the whole top carbon layer was eroded.

\subsection{Results of LIA-QMS}

Figure 6 shows the concentrations of $\mathrm{H}$ at different sample positions measured by LIA-QMS. Each LIA-QMS hydrogen signal in figure 6 consists of 5 sequential laser pulses (0 0.4 $\mu \mathrm{m}$ in depth). Higher integrated hydrogen contents were observed for the uncoated part of the tiles (without Mo and C layers), which are marked in light green. However, these higher $\mathrm{H}$ contents are not observed around the high erosion region (sample \#108), the hydrogen contents are almost the same between the maker area and the bulk graphite area in this region. The reduction of hydrogen retention in this high erosion region was caused by the higher surface temperature in this region (compared to the left and right part of the high erosion region), which is shown in figure 7.

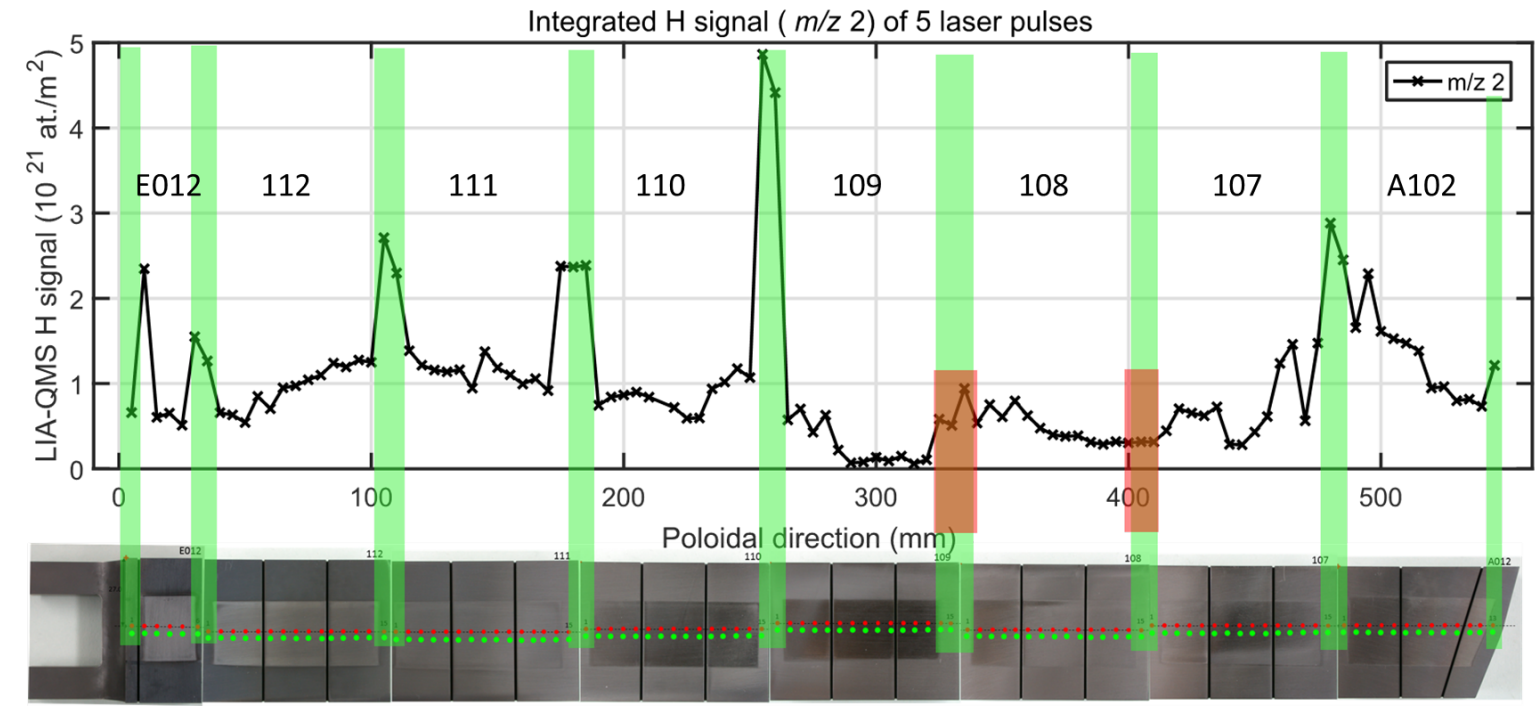

Figure 6. Distribution of $\mathrm{H}$ in poloidal direction. The green marked areas represent the bulk graphite areas, which are not covered with a marker layer. A higher concentration of $\mathrm{H}$ is observed in these areas. Meanwhile, a low $\mathrm{H}$ content is observed for the erosion dominated parts (marked in red).

\subsection{Heat flux and PFC erosion}

The erosion results were also compared to results of heat flux pattern measurements in $\mathrm{W} 7-\mathrm{X}$ for the standard divertor configuration [12]. The surface temperature was 
measured by infrared cameras. The heat flux pattern varies with plasma conditions and configurations, the pattern in figure 7 was obtained in discharge number of 20171108_040, and the detection time is 1s. As shown in figure 7, the heat flux pattern in a standard discharge configuration was inhomogeneous on the analyzed tiles. The peak heat flux is highest around the strike line in poloidal direction $(y=0.1$ $0.2 \mathrm{~m}$ ) in TM2h6, which matches well with the erosion dominated area with low fuel content determined by LIBS and LIA-QMS (red dot line marked area). The highest particle flux is also associated with the highest surface temperature [12]. The relationship between erosion and heat flux proves, a higher particle flux introduces higher temperature and more erosion, simultaneously.

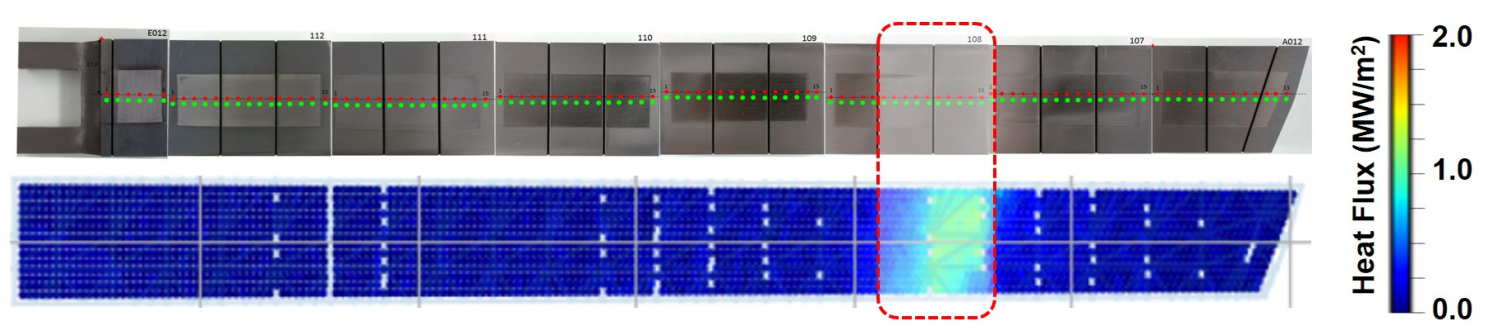

Figure 7. Comparison between the heat flux pattern and the erosion distribution of TM2h6 [12]. The position of the red box is same as figure 5 .

\section{Conclusion}

In this work, 8 graphite tiles from the divertor of W7-X were analysed in poloidal direction. To obtain the profile of various elements, a ps-laser was used to ablate samples pulse by pulse. Net Erosion and redeposition areas on these divertor tiles were identified through the depth distribution of the Mo spectra. The results show strong erosion of at least 5-10 $\mu \mathrm{m} \mathrm{C}$ and even the whole Mo layer at the strike line of the divertor. Some redeposition of Mo on the bulk graphite area of these tiles is observed. Meanwhile, lower $\mathrm{H}$ contents were observed around the erosion dominated area. The erosion profile on these samples was compared to the heat flux pattern during plasma operation in standard configuration, which shows a clear correlation. The presented analysis shows that the combination of ps-LIBS and LIAQMS can be used to identify erosion and redeposition areas and determine implantation of $\mathrm{H}$ on divertor tiles with marker efficiently, which is of great interest to study the plasma-wall interaction in W7-X for further operation phases. More tiles will be analyzed to compare the 10 divertor modules of $\mathrm{W} 7-\mathrm{X}$ as well as quantify the 
eroded $\mathrm{C}$ and $\mathrm{W}$ and compare it with RBS measurements and feed into global erosion and deposition modelling in order to describe the material migration in W7-X [13].

\section{Acknowledgments}

This work has been carried out within the framework of the EUROfusion Consortium and has received funding from the Euratom research and training programme 20142018 and 2019-2020 under grant agreement No 633053. The views and opinions expressed herein do not necessarily reflect those of the European Commission. This project is supported by China Postdoctoral Science Foundation funded project 2018M643159.

\section{References}

[1] Pedersen T S et al 2017 Phys. Plasmas 24055503

[2] Dinklage A, et al 2018 Nature Phys. 141067

[3] Wolf R S et al 2018 Plasma Phys. Control. Fusion 61014037

[4] Dhard, C P et al 2017 Phys. Scr. T170 014010.

[5] Dhard C P et al 2018 Fus. Eng. Des. In press

[6] Mayer M et al. 2019 PFMC Conf. (Eindhoven)

[7] Philipps V et al 2013 Nucl. Fusion 53093002

[8] Oelmann J et al 2019 Nucl. Mater. and Energ. 18 153-158

[9] Xiao Q et al 2015 J. Nucl. Mater. 463 911-4

[10] Li C et al 2015 J. Nucl. Mater. 463 915-8

[11] Ruset C et al 2009 Fusion Eng. Des. 84 1662-1665

[12] Gao Y et al 2019 Nucl. Fusion 59066007

[13] Brezinsek S et al. 2019 PFMC Conf. (Eindhoven) 